\title{
Form perception: Some effects of brightness and motion
}

\author{
JAMES T. WALKER and ROGER D. SAUNDERS \\ University of Missouri-St. Louis, St. Louis, Missouri
}

\begin{abstract}
A solid white bow tie moving horizontally across a black background appears distorted when the observer fixates a stationary spot: The points on the trailing half of the bow tie develop prominent "fins," and the trailing half appears longer in the horizontal dimension than the leading half of the moving figure. Visually tracking the moving bow tie eliminates these effects. A black bow tie moving across a white background does not produce these distortions. These effects are due in part to the differential visual persistence of the narrower and wider portions of the moving white display.
\end{abstract}

Consider a solid white symmetrical bow tie presented against a black background, as in Figure $1 \mathrm{~A}$. If the bow tie moves horizontally from left to right while the observer fixates a stationary point on the background, then the bow tie appears distorted approximately as shown in Figure 1C. Prominent "fins" develop at the points on the trailing edge of the bow tie, and the points on the leading edge appear to thicken approximately as illustrated. In addition, the trailing half of the figure (on the left in this case) appears longer in the horizontal dimension than the leading half.

A stationary white bow tie also appears distorted if the observer visually tracks another object-the point of a pencil, for example-moving horizontally in front of the display. Here, the retinal motion of the stationary display is opposite to the external motion of the tracking target. This retinal motion, like that of a moving figure viewed with stationary fixation, also produces fins at the trailing edge of the white figure. However, saccadic eye movements across a stationary bow tie produce no distortion. Visually tracking a moving white bow tie eliminates the distortions that would otherwise be observed with stationary fixation.

A black bow tie moving across a white background produces little or no distortion. With a stationary black bow tie, neither saccadic eye movements nor visually tracking an object moving across the display produces the distortions described above.

The illusory shape of the white bow tie illustrated in Figure $1 \mathrm{C}$ results from the differential visual persistence of the wider and narrower portions of the figure as measured in the horizontal dimension. Visual persistence, the length of time that a stimulus remains visible after its offset, is inversely related to the prior duration of the

Some of these results were presented at the annual meeting of the Southern Society for Philosophy and Psychology, Fort Worth. Texas. April 8, 1982. The authors' mailing address is: Department of Psychology, University of Missouri-St. Louis, 8001 Natural Bridge Road. St. Louis, MO 63121.

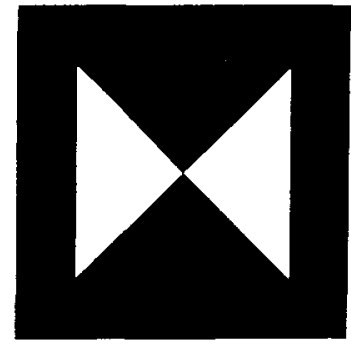

A.

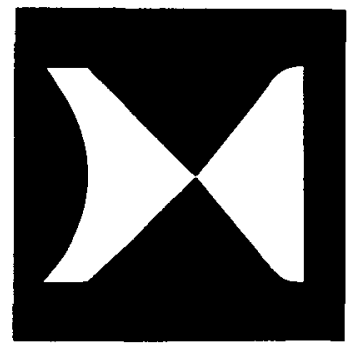

C.

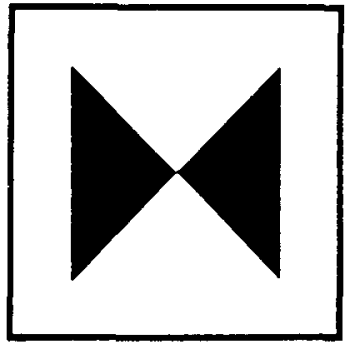

B.

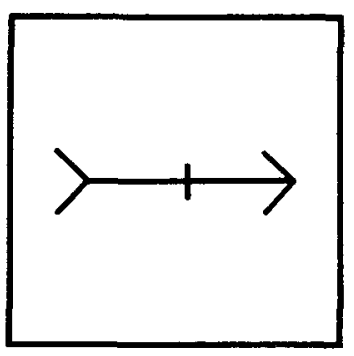

D.
Figure 1. (A) Stationary white bow tie against a black background. (B) Stationary black bow tie against a white background. (C) White bow tie moving from left to right appears distorted as shown. Trailing half, on the left here, appears longer horizontally than the leading half. (D) Modified Brentano form of the Müller-Lyer illusion.

stimulus-that is, the length of time the stimulus was present before offset (Bowen, Pola, \& Matin, 1974; Coltheart, 1980; Efron, 1970; Haber \& Standing, 1970; Walker \& Irion, 1982). As the image of a white bow tie sweeps horizontally across the retina, the narrower portions of the image - the regions near the points of the bow tie-are lighted for shorter durations than are the wider portions. Thus, the narrow points of the bow tie appear extended in the horizontal dimension-the points on the trailing half of the figure appear to develop fins, and the points on the leading half appear to thicken, as illustrated in Figure 1C. These distortions are much reduced in a 
white outline of a bow tie, as opposed to a solid figure, thus supporting the differential persistence hypothesis.

The resulting appearance of the white bow tie has some features in common with the Brentano form of the MüllerLyer illusion (Coren \& Girgus, 1978), a modified version of which is illustrated in Figure 1D. Thus, the fins that develop on the trailing half of the moving white bow tie might be expected to make that portion of the figure appear longer in the horizontal dimension than the leading half. However, the results of the present study do not support that hypothesis as an explanation of the observed lengthening.

The absence of distortion in the black bow tie requires explanation. As the black figure moves across the white background, all points in the retinal image are lighted by the background except during the brief passage of the black figure. Suppose, for example, that a black tie sweeps across the field of view once per second. In that case, each point in the retinal image is lighted for only a little less than a second, regardless of whether the illumination at any particular point is turned off by a wider or narrower portion of the black bow tie. Because all points in the retinal image are lighted for nearly the same relatively long period, there should be little or no difference in visual persistence between any portions of the black bow tie, and thus little distortion in the form of the moving black figure. This possible explanation, however, requires further consideration in light of the results of the present experiments.

Differential visual persistence also plays a role in another kinetic visual illusion, the bending hourglass (Walker, 1981). A slender white hourglass-essentially a narrow, vertically oriented bow tie-appears to bend as it moves across a black background (see Figure 2). If the observer fixates a stationary point, then the narrow middle portion of a moving white hourglass appears to thicken and to lag behind its true location. A black hourglass moving across a white background undergoes no such distortion. As discussed in a later section, the bending hourglass effect may have some bearing on the appearance of the moving bow ties.

In the present study, Experiment 1 measured the distortion in the apparent length of the leading and trailing

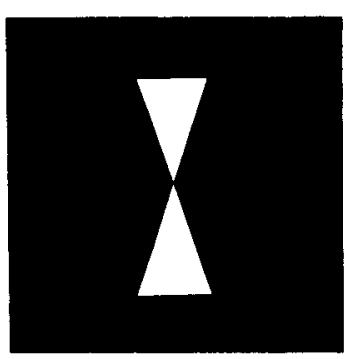

A.

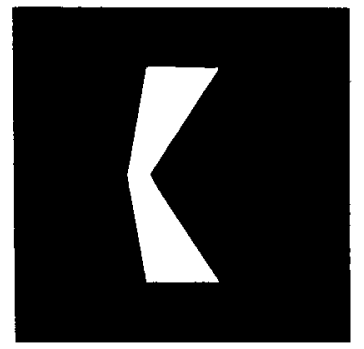

B.
Figure 2. (A) Stationary white hourglass against a black background. (B) Approximate appearance of an hourglass moving from left to right. halves of a moving white bow tie and demonstrated the absence of such distortion in a black figure. Experiments 2 and 3 assessed the possible role of the Müller-Lyer illusion in explaining that distortion. Experiment 4 measured the apparent length of the leading and trailing members of pairs of black and white bars in moving displays.

\section{EXPERIMENT 1}

This experiment measured the apparent length of the leading and trailing halves of moving black and white bow ties, using a magnitude estimation procedure.

\section{Method}

Subjects. Ten men and 10 women participated in the experiment. All were students in an undergraduate psychology course in research methods.

Apparatus. The displays were presented on a rotating vertical drum $15 \mathrm{~cm}$ in diameter and $17 \mathrm{~cm}$ in height. The drum was mounted on a tabletop a little below eye level at a viewing distance of $120 \mathrm{~cm}$. The bow ties were $100 \mathrm{~mm}$ in height and length, subtending visual angles of about $5^{\circ}$. The black bow tie was presented against a white background, and the white bow tie against a black background. A projector powered by a constant-voltage transformer lighted the white portions of the display at a luminance of $10 \mathrm{~mL}$ and the black portions at $0.28 \mathrm{~mL}$ as measured by a Macbeth illuminometer.

A black fixation spot, $6 \mathrm{~mm}$ in diameter, was projected onto the center of the rotating drum to serve as a stationary fixation point. The spot was formed by an opaque dot on a piece of clear glass in the slide plane of the projector, and was clearly visible on the black as well as the white background of the display. The experimental room was dark except for stray light from the projector.

Procedure. To control for possible individual differences in the apparent size of objects in the left and right visual fields (Brown, 1953), each subject judged the apparent length in the horizontal dimension of the left and right halves of the stationary black and white bow ties. The subject was instructed to fixate the black spot and to indicate whether the left or right portion of the display appeared longer, and to judge in percentage terms how much longer it appeared. Judgments of equality were permitted.

Each subject then viewed the black and white bow ties moving at one revolution per second, yielding a linear velocity of about $22^{\circ}$ of visual angle per second at the center of the display. Blackwhite and white-black orders were balanced. Each bow tie was presented for two trials, once moving to the left and once to the right. Directions of movement were randomized for each subject. The subject fixated the stationary black spot as steadily as possible and then indicated whether the front or rear portion (leading or trailing) looked longer in the horizontal dimension. As in the control judgments, the subject then indicated in percentage terms how much longer that portion of the display looked. Subjects required periods varying from a few seconds to a few tens of seconds to make their judgments.

\section{Results and Discussion}

The data were subjected to a three-way analysis of variance in which the factors were color of bow tie (black, white), sequence of colors (black-white, white-black), and direction of motion (left, right). There were repeated measures on the color and direction factors. Only the effect of color was significant $[F(1,18)=14.44, p<.01]$; all other $F$ ratios were less than 1 .

Because there was no significant effect of direction of motion, the two directions were pooled. For the black bow 
tie, the pooled mean and standard deviation were $1.70 \%$ and $11.15 \%$; that mean did not differ significantly from zero $[\mathrm{t}(19)<1]$. For the white bow tie, the pooled mean and standard deviation were $15.95 \%$ and $15.43 \%$; the mean here differed significantly from zero $[\mathrm{t}(19)=4.62$, $\mathrm{p}<.001]$.

As described above, the moving white bow tie develops fins that can be expected to make the trailing half of that figure appear longer, much as the outward-pointing fins influence distance judgments in the Brentano form of the Müller-Lyer illusion (see Figure 1D). But the black bow tie develops no such fins. Thus, if the fins on the white bow tie are responsible for the perceived difference in length between the leading and trailing halves, then only the white bow tie should appear distorted in that way, and the black bow tie should not. The results of the present experiment are consistent with these expectations.

\section{EXPERIMENT 2}

This experiment tested a form of the Müller-Lyer illusion as a possible factor in the distortions of apparent length observed in the moving white bow tie. Several graded series of stationary displays were measured, using a magnitude estimation procedure.

\section{Method}

Subjects. Five women and 3 men, undergraduate psychology students at the University of Missouri-St. Louis, received extra course credit for their participation. All subjects claimed to be free of any significant visual problems. One subject was replaced for the reason described in the Procedure section.

Materials and Apparatus. Stationary displays were designed to represent the essential features of the moving white bow tie, as in Figure 1C, which depicts the appearance of a white bow tie moving from left to right. That display will be described as a rightfacing illusion figure. A left-facing illusion figure, depicting the appearance of a white bow tie moving from right to left, would be rotated $180^{\circ}$

All of the illusion figures were $100 \mathrm{~mm}$ in height, and each figure was presented individually in a separate display. Four graded series of illusion figures were prepared: left-facing black figures, leftfacing white figures, right-facing black figures, and right-facing white figures. The measurements in millimeters of the horizontal dimensions of the left and right portions of the figures were as follows for the four series, respectively: L60-R50, L55-R50, L50R55, L50-R60. A series of pairs of white bars with the same horizontal dimensions as the illusion figures described above were mounted on black backgrounds, and a series of pairs of black bars were mounted on white backgrounds. Each bar was $20 \mathrm{~mm}$ in height, and the bars were $20 \mathrm{~mm}$ apart within each pair.

All of the displays were front-lighted by the same projector powered by a constant-voltage transformer. The luminance of the white figures was $10 \mathrm{~mL}$, and the luminance of the black background was $0.42 \mathrm{~mL}$. The luminance of the black figures was $0.10 \mathrm{~mL}$, and the luminance of the white background was $9.8 \mathrm{~mL}$. The displays were presented at about eye level in a frontoparallel plane at a viewing distance of $120 \mathrm{~cm}$. Thus, the displays subtended a visual angle of about $5^{\circ}$, as in Experiment 1 .

Procedure. There were six categories of displays: black illusion figures, left- and right-facing; white illusion figures, left- and rightfacing; pairs of black bars; and pairs of white bars. Each category contained 4 displays, measuring L60-R50, L55-R50, L50-R55, and L50-R60, as above. Thus, there were 24 displays in all. Each sub- ject viewed all of these displays in an individually randomized sequence.

The subjects indicated whether the left or the right portion of each display looked longer, and then judged that portion in relation to the shorter appearing portion. Half the subjects made their judgments in millimeters, using the $50-\mathrm{mm}$ length as a standard. The other subjects made percentage estimates of the longer appearing portion in relation to the shorter appearing. One subject, who responded to every display with a judgment of $25 \%$ or $33 \%$ longer, was replaced because of that response bias.

\section{Results and Discussion}

The judgments in millimeters were converted to percentages and pooled with the judgments originally made in percentages. If an illusion occurs, then the left portion of a right-facing illusion figure should appear longer (see Figure 1B), and so should the right portion of a left-facing figure. Responses supporting the occurrence of an illusion were considered positive, and nonsupporting responses were considered negative. To control for possible left-right hemifield differences in perceived size (Brown, 1953), judgments of the black and white bars were subtracted from the judgments of the illusion figures. The results from the left-facing and right-facing illusion figures were then combined.

The means for the black and white illusion figures did not differ significantly $(t<1)$, and thus were pooled. The mean and standard deviation for the combined black and white figures were $0.67 \%$ and $0.87 \%$. The combined mean differed significantly from zero by a one-tailed test $[\mathrm{t}(7)=2.18, \mathrm{p}<.05]$.

The very small magnitude of the observed illusion $(0.67 \%)$, although in the expected direction, cannot account for the $15.95 \%$ distortion in the moving white bow tie measured in Experiment 1. Thus, these results do not support the Müller-Lyer hypothesis advanced in Experiment 1 to explain the apparent lengthening of the trailing portion of the moving white bow tie.

\section{EXPERIMENT 3}

This experiment further investigated the possible role of the Müller-Lyer illusion as a factor in the distortion of the moving white bow tie in Experiment 1. Experiment 3 used an adjustment method to measure the apparent length of the left and right portions of a stationary white illusion figure.

\section{Method}

Subjects. Eight men and 8 women, undergraduate psychology students, received extra course credit for their participation. No subjects were replaced because of visual problems, although one subject reported a diagnosis of amblyopia.

Apparatus. The illusion figure was cut out of a black mask mounted in front of a translucent white Plexiglas sheet backlighted by a projector powered by a constant-voltage transformer. The luminance was $10 \mathrm{~mL}$ on the viewing side of the display. The room was dark except for stray light from the projector. The length of the finned portion of the display (the left portion in Figure 1C) was adjusted in the horizontal dimension by a knob available to the subject's preferred hand. Thus, the finned portion of the display was the adjustable comparison stimulus and the other portion was the 
standard stimulus. The illusion figure was $100 \mathrm{~mm}$ in height, and the horizontal length of the standard portion (the right portion in Figure 1C) was $50 \mathrm{~mm}$.

Procedure. All subjects were shown the illusion figure facing left on half the trials and facing right (as in Figure 1C) on the other half. Left-right presentation orders were counterbalanced across subjects. The subjects were given four trials on each illusion figure, half of the subjects in ADDA order and half in DAAD order. The sexes were balanced with respect to all of those conditions. At the start of each ascending or descending trial, the comparison was set $10 \mathrm{~mm}(20 \%)$ shorter or longer than the standard. The experimenter pointed out the horizontal dimensions of the left and right portions of the display and instructed each subject to adjust the comparison so that it appeared the same length as the standard.

\section{Results and Discussion}

The data were subjected to a repeated-measures Latin square analysis of variance. The factors were sex, orientation (left-facing, right-facing), and temporal order (first, second). There were repeated measures on the last two factors.

The effect of sex approached significance $[F(1,12)=$ $3.63, p<.10]$. There was no significant effect of temporal order $[F(1,12)=1.44, p>.25]$, nor was there any other significant effect [all other Fs $<1$ ]. Pooling the results for men and women across orientations and temporal orders, the combined mean of 2.09 was not significantly different from zero $[t(15)=1.32]$.

The results of Experiment 3 are essentially consistent with those of Experiment 2, in that the small magnitude of illusion in the stationary displays cannot account for the substantially larger distortion observed in the moving white bow tie in Experiment 1. Thus, the Müller-Lyer hypothesis offered in Experiment 1 is untenable in light of the subsequent two experiments.

\section{EXPERIMENT 4}

If the finned appearance of the moving white bow tie has little or no effect on the apparent lengthening of the trailing half of the figure-as Experiments 2 and 3 suggest-then it may be that any trailing white stimulus will appear longer than a leading stimulus of the same objective length. Experiment 4 addressed this possibility by measuring the apparent length of leading and trailing members of pairs of black and white bars.

\section{Method}

Subjects. Eight men and 8 women, undergraduate psychology students, received extra course credit for their participation. All subjects claimed to be free of significant visual problems.

Apparatus. The same apparatus and the same levels of luminance were used as in Experiment 1. Two moving displays were used: a pair of solid black bars against a white background, and a pair of solid white bars against a black background. The bars were 20 $\times 50 \mathrm{~mm}$, were oriented horizontally, and were separated by $20 \mathrm{~mm}$ within each pair. The drum rotated to the left or right at one revolution per second, yielding a linear velocity of about $22 \% \mathrm{sec}$ at the viewing distance of $120 \mathrm{~cm}$, as in Experiment 1 .

A graded series of comparison stimuli was presented in a frontoparallel plane immediately above the center of the drum (see
$3 L$
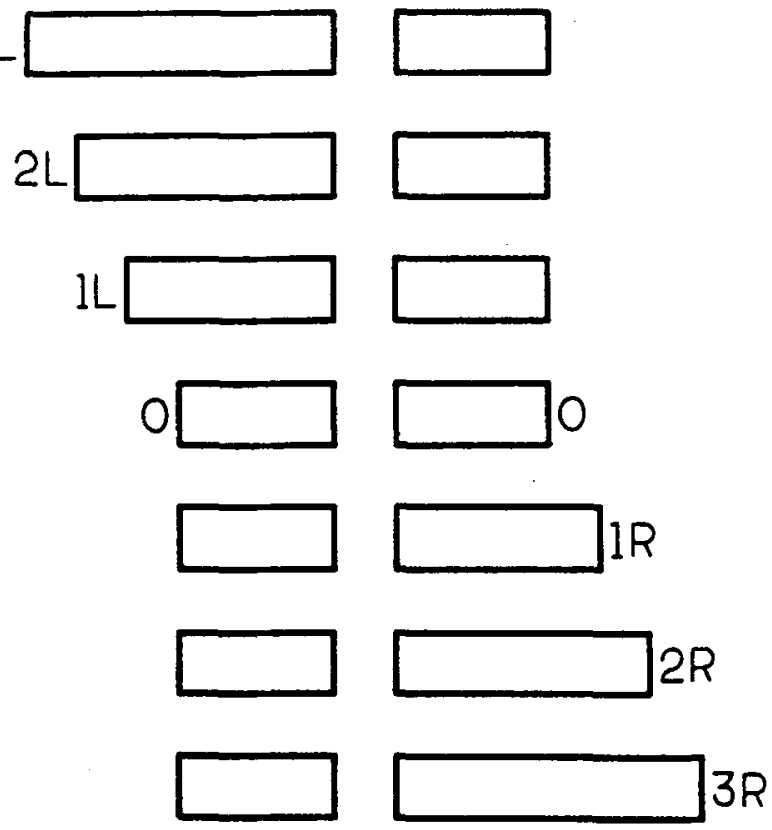

Figure 3. Comparison stimuli in Experiment 4.

Figure 3). The comparison stimuli were pairs of outline bars, black lines against a white background with a luminance of $10 \mathrm{~mL}$. The bars were $20 \mathrm{~mm}$ in height. The shorter member of each pair was $50 \mathrm{~mm}$ long, and the longer member was $33.3 \%, 66.6 \%$, or $100 \%$ longer.

Procedure. As in Experiment 1, a black fixation point was projected onto the center of the drum. Control measures were obtained by presenting the stationary displays. The subjects fixated the black spot and then selected the pair of comparison stimuli that appeared most similar to the pair of bars on the drum. The drum was then rotated left or right. The subjects judged the moving bars while fixating the black spot, and then chose the comparison pair that appeared most similar to the moving bars. Subjects were encouraged to make finer judgments in terms of tenths of the difference between adjacent comparison pairs. The subjects had no difficulty in following these procedures. Orders of black and white displays and directions of motion were counterbalanced, and the sexes were balanced across those conditions.

\section{Result and Discussion}

The results were subjected to an analysis of variance where the factors were sex, color of bars (black, white), and direction of motion (left, right). There were repeated measures on the last two factors. There was no significant effect of sex or direction of motion [Fs $<1$ ] or color of bars $[F(1,14)=2.96, p<.25]$, but the interaction between these three factors approached significance $[F(1,14)=4.36, p<.10]$.

Because there was no significant effect of direction of motion, the two directions were combined. For both the black and white displays, the trailing bar appeared longer. The mean and standard deviation of the apparent lengthening were $19.67 \%$ and $12.67 \%$, respectively, for the black bars, and $27.67 \%$ and $24.00 \%$ for the white bars. Both of these means were significantly different from zero 
[for black bars, $t(15)=6.21$; for white bars, $t(15)=$ 4.61, both ps $<.001]$.

The observed temporal order effect may be due to the foreshortening and disappearance of the leading bar around the side of the rotating drum while the trailing bar is still fully visible. Thus, the trailing stimulus is objectively longer in the retinal image, at least for a time. But as the bars move into view around the edge of the drum, the leading bar projects a longer image initially. It is not entirely obvious why the most recent comparison between the bars, in which the trailing bar appears longer, should dominate one's judgment of those stimuli. However, the present results are consistent with the temporal order or recency effect often observed in other situations, in which a comparison stimulus is overestimated in relation to a previously presented standard (see, e.g., Osgood, 1953).

The temporal order effect in the present experiment is more than sufficient to account for the greater apparent length of the trailing half of the white bow tie in Experiment 1 . But the trailing black bar also appeared longer here, and thus the trailing half of the black bow tie in Experiment 1 should also have appeared longer-contrary to the observed results of that experiment. These conflicting results can be reconciled by considering the bending hourglass effect.

A black bow tie against a white background partially encloses the central portion of a vertical white hourglass. Because the narrow portion of a moving white hourglass has been shown to lag behind the other portions of the figure (Walker, 1981), this effect would shorten the apparent length of the trailing half of a black bow tie, and would thus run counter to the temporal order effect observed in the present experiment. The net result would be to reduce or eliminate any apparent lengthening of the trailing half of the black bow tie that might otherwise be produced by the temporal order effect.

\section{GENERAL DISCUSSION}

In Experiment 1, a moving white bow tie displayed the distortions illustrated in Figure 1C, but a black bow tie did not. The trailing half of the white bow tie appeared longer than the leading half, but a black bow tie showed no such distortion. Although the finned appearance of the moving white bow tie shares some features of the MüllerLyer illusion, which might account for the apparent lengthening of the trailing half, Experiments 2 and 3 rendered the Müller-Lyer hypothesis untenable.

In Experiment 4, the trailing member of a pair of black or white bars appeared significantly longer than the leading member. This temporal order effect would be expected to make the trailing half of the black bow tie, as well as the white bow tie, appear longer in Experiment 1. However, the bending hourglass effect, as explained in the discussion of Experiment 4, would be expected to reduce or eliminate the temporal order effect that might otherwise occur in the black bow tie.

Brightness reversal-interchanging the light and dark portions of a visual stimulus, as in the black and white figures in the present study-preserves all of the information in the visual display. Nevertheless, lightness and darkness play asymmetrical roles in the present context, and in some other perceptual situations as well. For example, it is difficult to recognize a familiar face in a blackand-white photographic negative, or negative print, even though such a display contains the same information as a conventional positive print (Galper, 1970).

Black and white bow ties contain the same visual information, in the sense of form and contour, but these figures appear very different in motion. In this respect, the present phenomena are similar to the bending hourglass described earlier. The fins that develop on the white bow tie, and the bending of the white hourglass, are explained in terms of the greater visual persistence of the narrower portions of the moving white displays. The $a b-$ sence of such effects in the black bow tie and the black hourglass is consisent with this explanation. The present study offers further evidence of the asymmetry between lightness and darkness in visual form perception.

\section{REFERENCES}

Bowen, R. W., Pola, J., \& Matin, L. (1974). Visual persistence: Effects of flash luminance, duration, and energy. Vision Research, 14, 295-303.

Brown, K. T. (1953). Factors affecting differences in apparent size between opposite halves of a visual meridian. Journal of the Optical Society of America, 43, 464-472.

Coltheart, M. (1980). Iconic memory and visible persistence. Perception \& Psychophysics, 27, 183-228.

Coren, S., \& GirGus, J. S. (1978). Seeing is deceiving: The psychology of visual illusions. Hillsdale, NJ: Erlbaum.

EFron, R. (1970). Effects of stimulus duration on perceptual onset and offset latency. Perception \& Psychophysics, 8, 231-234.

GALPER, R. E. (1970). Recognition of faces in photographic negatives. Psychonomic Science, 19, 207-208.

Haber, R. N., \& Standing, L. G. (1970). Direct estimates of the apparent duration of a flash. Canadian Journal of Psychology, 24, 216-229.

OSGOOD, C. E. (1953). Method and theory in experimental psychology. New York: Oxford University Press.

WALKER, J. T. (1981). A new dynamic visual illusion: The bending hourglass. Perception \& Psychophysics, 30, 455-458.

WALKER, J. T., \& IRION, A. L. (1982). Apparent displacement of moving and stationary strobe flashes. Human Factors, 24, 213-224.

(Manuscript received April 15, 1985; revision accepted for publication October 11,1985 .) 